\title{
A Compact Size Reconfigurable Antenna for Ultra-Wideband Applications
}

\author{
Manisha Mishra ${ }^{1}$, Juhi Priyani ${ }^{2}$, Abhijeet Dasgupta ${ }^{3}$ \\ 1, 2, 3 (Amity School of Engineering and Technology, Amity University, Noida, Uttar Pradesh-201303)
}

\begin{abstract}
This paper presents a simpler method of designing a compact size reconfigurable antenna useful for ultra-wideband applications. The reconfigurability of the antenna designed was achieved by using the concept of open-circuit and short-circuit so only single structure was designed and reconfigurability of frequency was achieved. The substrate on which the antenna was to be designed was taken as Taconic(TLY) and the ground part was taken as Copper.The feed was given through the lumped port at $18 \mathrm{~mm}$ in the XZ plane from the origin of the 3-dimensional co-ordinates i.e the starting left point of the substrate. Due to reconfigurable ability of the antenna four bands were obtained namely band-I (7.1GHz-10.8GHz), band II (7.3GHz-11.3GHz.), band III $(12.8 \mathrm{GHz}-15.6 \mathrm{GHz}) \&$ band-IV $(17.3 \mathrm{GHz}-18.8 \mathrm{GHz})$ with corresponding return losses of $-33,-25.4$ and -25.9 dB respectively.
\end{abstract}

Keywords: compact size antenna, microstrip patch, reconfigurability, ultra-wideband applications, cognitive radio applications

\section{INTRODUCTION}

Reconfigurable antenna reveals various beneficial compared to the conventional antenna at the same class. It achieved multi function for a similar antenna. Moreover, the antenna size and cost can be minimized contrast to the separate task of the conventional antenna. The application of reconfigurable antenna is not only limited for the surveillance, but it is reliable for the military communication as well. The dynamically reconfigurable antenna can be comprehended via RF switches such as PIN diodes, MEMs and GaAs FETs. By changing the switch state to either 'ON' or 'OFF' mode, these elements capable for frequency tuning [1-5] and practical for impedance matching devices [6-7]. This paper introduces the concept of frequency reconfigurable antenna bandwidth control and the merit of using frequency-reconfigurable antennas. The design of reconfigurable antennas has received a lot of attention in recent years. With the increase in demand of bandwidth, enormous amount of efforts has been laid to the development of antennas that can function on more than one band. The biggest challenge that lies in the design methodology adopted for reconfigurable antennas is to connect the radiating elements together such that the resulting structure will give us the required RF response over the bands of interest. The most common methodology adopted for reconfigurable antenna design is the inclusion of some form of switching circuitry. In the past, switches have been incorporated using RF-MEMS, pi-n diodes, or lumped elements. Micro-electro mechanical systems (MEMS) switch Despite all their advantages, the use of these switching elements requires the design of an appropriate biasing network for the activation/deactivation of the switch, which can affect the antenna performance and add further complexity to the antenna structure. In this paper PIN diode switches has been incorporated in the patch network.

\section{ANTENNA CONFIGURATION}

The substrate on which the antenna was to be designed was taken as Taconic(TLY) and the ground part was taken as Copper. The feed was given through the lumped port at $18 \mathrm{~mm}$ in the $\mathrm{XZ}$ plane from the origin of the 3-dimensional co-ordinates i.e the starting left point of the substrate. The substrate shape is a square of size $38 \mathrm{~mm} \times 38 \mathrm{~mm} \times 1.05 \mathrm{~mm}$. The dimensions of the ground plane are $38 \mathrm{~mm} \times 38 \mathrm{~mm} \times 0.035 \mathrm{~mm}$.

The dimensions of the square and rectangle shaped patch are shown in Fig. 1 below:
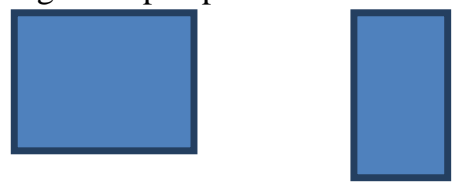

Figure 1: Dimensions Of the patches

The Figure below shows the schematic of the reconfigurable antenna designed in Ansoft HFSS. The antenna designed consists of air box on which a square shaped substrate is placed. The individual square shaped patches are arranged in the fashion shown below which is the configuration when all the switches are in closed 
fashion. For making the circuit shown below in Fig. 2 as reconfigurable, the concept of open and short circuit has been used. Same material was used to form an open circuit when closing of switch was required as it was used in patch and hence reconfigurablity of frequency was achieved.

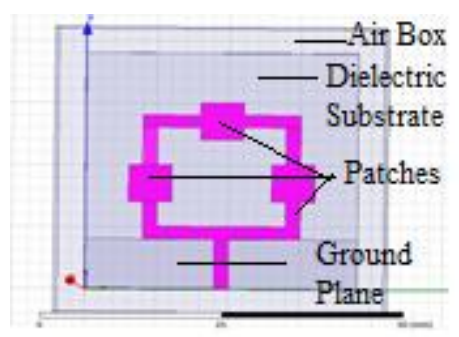

Figure 2: Antenna configuration when all the switches are closed

A configuration of the antenna when one of the patches is removed is shown in the Fig. 3 below

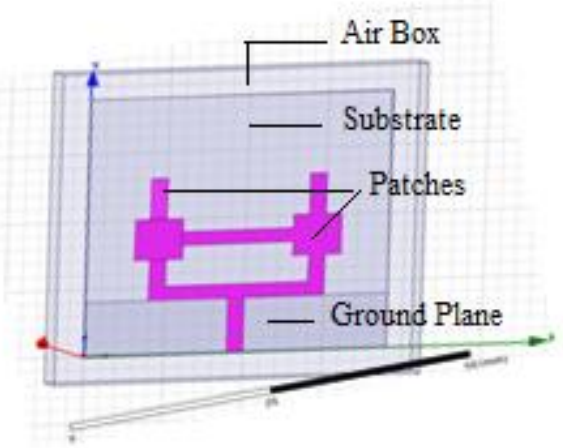

Figure 3: Antenna configuration when left and right patches are connected and top patch is removed

In the third configuration, the left side rectangular patch is removed and then its results are studied.

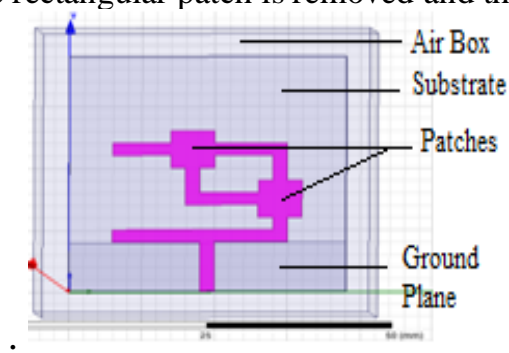

Figure 4: Configuration of antenna when top patch and right patch are connected

\section{RESULTS AND DISCUSSIONS}

With the designing of different configurations, frequency reconfigurability was achieved over four bands that is band-I $(7.1 \mathrm{GHz}-10.8 \mathrm{GHz})$, band II $(7.3 \mathrm{GHz}-11.3 \mathrm{GHz}$.), band III $(12.8 \mathrm{GHz}-15.6 \mathrm{GHz}) \&$ band-IV $(17.3 \mathrm{GHz}-18.8 \mathrm{GHz})$ with corresponding return losses of $-33,-25.4$ and $-25.9 \mathrm{~dB}$ respectively. The below table shows the operating frequency, bandwidth and return loss of the designed antenna in different configurations.

Table 1: Operating frequency, bandwidth and return loss of the designed antenna

\begin{tabular}{|l|c|c|c|}
\hline CONFIGURATION & $\begin{array}{l}\text { FREQUENCY } \\
\text { BAND(GHz) }\end{array}$ & BANDWIDTH(GHz) & $\begin{array}{l}\text { RETURN } \\
\text { LOSS(dB) }\end{array}$ \\
\hline Config-1 & $\mathbf{7 . 1}-\mathbf{1 0 . 8}$ & $\mathbf{3 . 7}$ & $\mathbf{- 3 3}$ \\
\hline Config-2 & $\mathbf{1 2 . 8 - 1 5 . 6}$ & $\mathbf{2 . 8}$ & $\mathbf{- 2 5 . 4}$ \\
\hline Config-3 & $\mathbf{7 . 3}-\mathbf{1 1 . 3}$ & 4 & $-\mathbf{2 5 . 9}$ \\
\hline
\end{tabular}




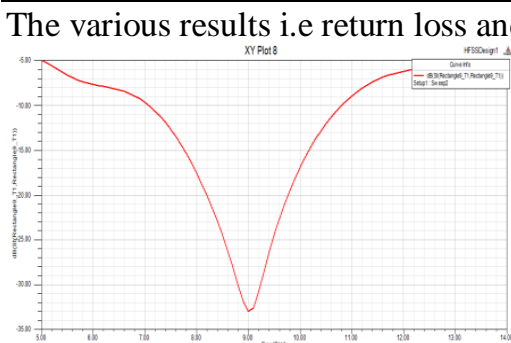

Figure 5: Return loss corresponding to first configuration

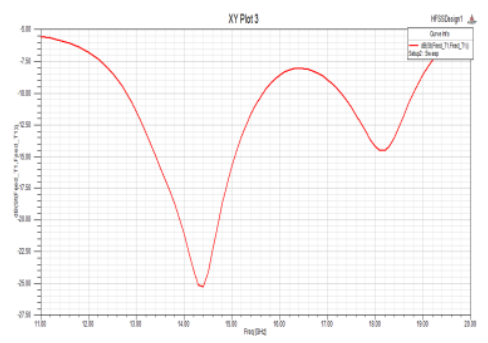

Figure 6: Return loss corresponding to second configuration

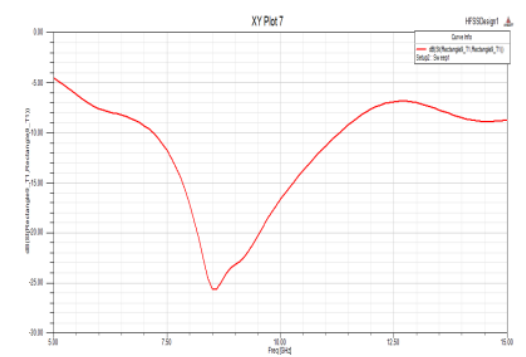

Figure 7: Return loss corresponding to third configuration

The maximum radiation direction of the antenna, gain and polarization plot obtained corresponding to maximum return loss of $-33 \mathrm{~dB}$ is as follows

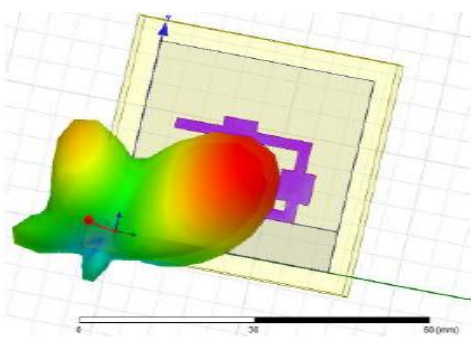

Figure 11: Direction Of Maximum Radiation

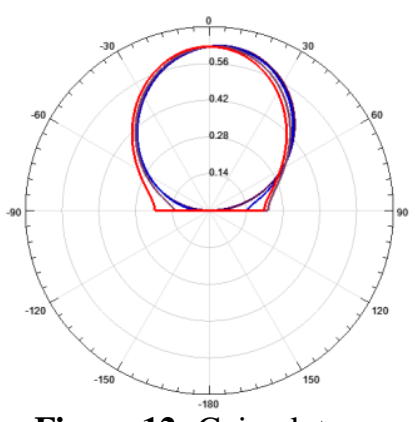

Figure 12: Gain plot corresponding
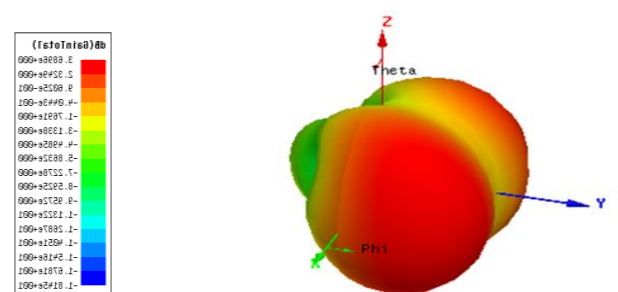

Figure 8: Radiation pattern of first config.
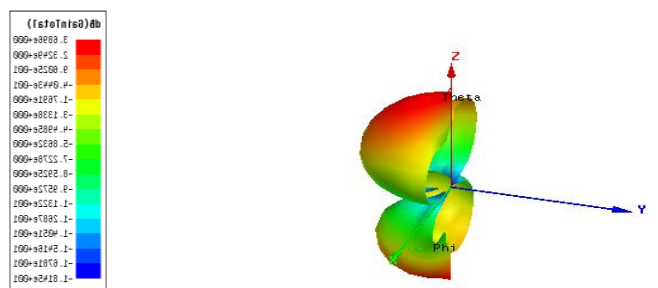

Figure 9: Radiation pattern of second config.
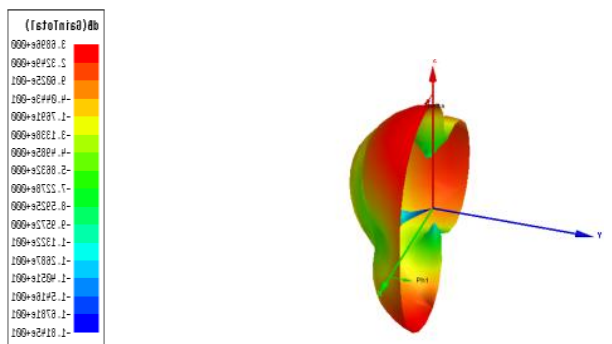

Figure 10: Radiation pattern of third config.

PolarizationRatio to maximum return loss

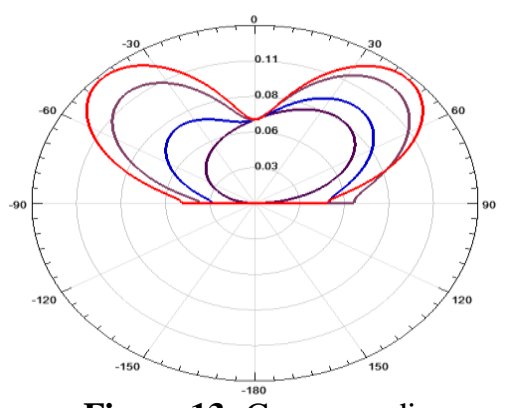

Figure 13: Corresponding 


\section{CONCLUSIONS}

A compact size frequency reconfigurable antenna has been designed which can be tuned to different frequencies to achieve desired frequency band for different applications. The return loss and radiation pattern(gain plot) has been studied under various configurations..The maximum return loss of $-33 \mathrm{~dB}$ has been achieved under the condition when all the patches are kept intact that is under first configuration. The traditional antenna covering several frequency bands concurrently with a single antenna having enough efficiency and bandwidth is a major challenge. One possible solution to this problem is the use of reconfigurable antennas that tune to different frequency bands. Such an antenna would not cover all bands simultaneously, but provides narrower instantaneous bandwidths that are dynamically selectable at higher efficiency than conventional antenna. The designed antenna can be widely used in ultra-wideband applications and small wireless networks. In future, this reconfigurable antenna can be extended for MIMO applications and for satellite and terrestrial links.

\section{References}

[1] M.R. Hamid, P. Gardner, P.S. Hall, F. Ghanem, "Switchable Wideband-Narrowband Tapered Slot Antenna," Loughborough Antennas \& Propagation Conference. 16-17 November 2009, Loughborough, UK, 241-244, 2009.

[2] Qiang Chen, Makoto Kurahashi and Kunio Sawaya, "Dual-mode Patch Antenna Switched by PIN Diode," IEEE Topical Conference on Wireless Communication Technology. 148-149, 2003.

[3] S. V. Shynu, G. Augustin, C. K. Aanandan, P. Mohanan, and K. Vasudevan, "Design of Compact Reconfigurable Dual Frequencies Microstrip Antennas Using Varactor Diodes,” Progress in Electromagnetic Research, PIER 60, 197-205, 2006.

[4] F. Ghanem, J.R. Kelly, and P.S. Hall, "Switched UWB to Narrowband Planar Monopole Antenna," European Conference on Antennas and Propagation 2010, 12-16 April 2010, Barcelona.

[5] M. T.Ali, T. A. Rahman, M.R. Kamarudin and M. N. Md Tan, "Reconfigurable Linear Array Antenna with Beam Shaping at 5.8GHz," Microwave Conference, 2008. APMC 2008. Asia-Pacific.

[6] M. A. Saed, "Reconfigurable Broadband Microstrip Antenna fed by a Coplanar Waveguide," Progress In Electromagnetics Research, PIER 55, 227-239, 2005.

[7] M. F. Jamlos, O. A. Aziz, T. A. Rahman, M. R. Kamarudin, P. Saad, M. T. Ali and M. N. Md Tan, "A Beam Steering Radial Line Slot Array (RLSA) Antenna with Reconfigurable Operating Frequency," J. of Electromagn. Waves and Appl., Vol. 24, 1079-1088, 2010. 